\title{
Numerical Analysis of Ridged-Circular Nanoaperture for Near-Field Optical Disk
}

\author{
Toshiaki Kitamura \\ Faculty of Engineering Science, Kansai University, Suita, Osaka 564-8680, Japan \\ Correspondence should be addressed to Toshiaki Kitamura; kita@kansai-u.ac.jp
}

Received 22 August 2013; Accepted 19 September 2013

Academic Editors: J. M. Gonzalez-Leal and L. Kassab

Copyright (C) 2013 Toshiaki Kitamura. This is an open access article distributed under the Creative Commons Attribution License, which permits unrestricted use, distribution, and reproduction in any medium, provided the original work is properly cited.

\begin{abstract}
A ridged-circular nanoaperture is investigated through three-dimensional (finite-difference time-domain) FDTD method. The motion equations of free electrons are inserted to analyze a metallic material. The electromagnetic field distributions of optical near-field around the aperture are investigated. The phase change disk illuminated by a near-field optical light through a ridgedcircular nanoaperture is also analyzed. The far-field scattering patterns from the phase change disk and the crosstalk characteristics between plural marks are studied.
\end{abstract}

\section{Introduction}

The recording density of conventional optical recording systems has an optical diffraction limit. Recently, there has been a lot of interest in the field of optical storage technologies for the recording methods that are based on near-field optical principles. This is because they have the potential to overcome the limitation by using the localized optical nearfield for writing and reading recorded marks. Many types of nanoapertures and nanoantennas have been proposed to achieve the high throughput of the optical near-field [14]. This study focuses on a ridged-circular nanoaperture for a near-field optical disk. The analysis is accomplished by the FDTD method into which the motion equations of free electrons are inserted [5-7]. This method can easily deal with Drude dispersion and it can be applied to the analysis of various plasmonic devices. First, the electromagnetic field distributions of optical near-field around the ridged-circular nano-aperture are analyzed. Next, the scattering characteristics from a phase change disk with the aperture are studied. Finally, the crosstalk characteristics between plural marks are discussed.

\section{FDTD Formulation}

In the FDTD method, special handling of the metallic material is required because the permittivity is dispersive and has a negative value in the optical frequency. In this study, the following motion equation is introduced into the FDTD method to evaluate the conducting current:

$$
m \frac{d \mathbf{u}}{d t}=-e \mathbf{E}-m \nu \mathbf{u},
$$

where $\mathbf{u}$ is the electron velocity, $\mathbf{E}$ is the electric field, $e$ is the elementary electric charge, $m$ is the electron mass, and $v$ is the collision frequency. The collision frequency is expressed as follows:

$$
\nu=\frac{2 n_{1} n_{2} \omega}{1-n_{1}^{2}+n_{2}^{2}},
$$

where $\omega$ is the angular frequency of a light wave and $n_{1}$ and $n_{2}$ are the real and imaginary parts, respectively, of the complex refractive index $N$ of a metallic material $\left(N=n_{1}-j n_{2}\right)$.

Maxwell's equations are expressed as follows by representing the current density using the electron velocity $\mathbf{u}$ and the electron density $n_{0}$ :

$$
\begin{gathered}
\varepsilon_{0} \frac{\partial \mathbf{E}}{\partial t}=\nabla \times \mathbf{H}+n_{0} e \mathbf{u}, \\
\mu_{0} \frac{\partial \mathbf{H}}{\partial t}=-\nabla \times \mathbf{E},
\end{gathered}
$$

where $\mathbf{H}$ is the magnetic field and $\varepsilon_{0}$ and $\mu_{0}$ are the electric permittivity and magnetic permeability of free space, respectively. 


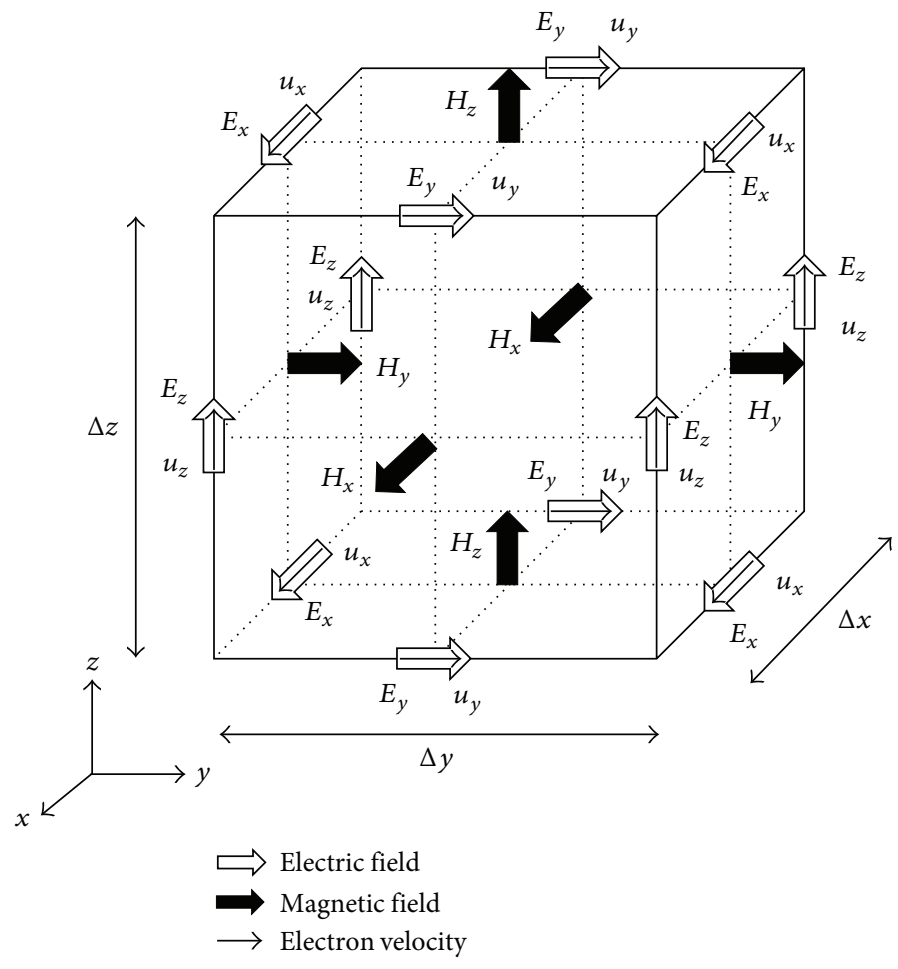

FIgURE 1: FDTD cell for analysis.

Figure 1 shows the FDTD cell for analysis. The electron velocity components $u_{x}, u_{y}$, and $u_{z}$ are introduced and arranged at the same positions as $E_{x}, E_{y}$, and $E_{z}$, respectively. By evaluating the electron velocity components at the same time as the magnetic field components, the finite difference equations corresponding to (1), (3) are expressed as follows:

$$
\begin{gathered}
E_{x}^{n}=E_{x}^{n-1}+\frac{\Delta t}{\varepsilon_{0}}\left(\frac{\partial H_{z}^{n-1 / 2}}{\partial y}-\frac{\partial H_{y}^{n-1 / 2}}{\partial z}\right)+\frac{\Delta t}{\varepsilon_{0}} n_{0} e u_{x}^{n-1 / 2}, \\
E_{y}^{n}=E_{y}^{n-1}+\frac{\Delta t}{\varepsilon_{0}}\left(\frac{\partial H_{x}^{n-1 / 2}}{\partial z}-\frac{\partial H_{z}^{n-1 / 2}}{\partial x}\right)+\frac{\Delta t}{\varepsilon_{0}} n_{0} e u_{y}^{n-1 / 2}, \\
E_{z}^{n}=E_{z}^{n-1}+\frac{\Delta t}{\varepsilon_{0}}\left(\frac{\partial H_{y}^{n-1 / 2}}{\partial x}-\frac{\partial H_{x}^{n-1 / 2}}{\partial y}\right)+\frac{\Delta t}{\varepsilon_{0}} n_{0} e u_{z}^{n-1 / 2}, \\
H_{x}^{n+1 / 2}=H_{x}^{n-1 / 2}-\frac{\Delta t}{\mu_{0}}\left(\frac{\partial E_{z}^{n}}{\partial y}-\frac{\partial E_{y}^{n}}{\partial z}\right), \\
H_{y}^{n+1 / 2}=H_{y}^{n-1 / 2}-\frac{\Delta t}{\mu_{0}}\left(\frac{\partial E_{x}^{n}}{\partial z}-\frac{\partial E_{z}^{n}}{\partial x}\right), \\
H_{z}^{n+1 / 2}=H_{z}^{n-1 / 2}-\frac{\Delta t}{\mu_{0}}\left(\frac{\partial E_{y}^{n}}{\partial x}-\frac{\partial E_{x}^{n}}{\partial y}\right), \\
u_{x}^{n+1 / 2}=\frac{2-v \Delta t}{2+\nu \Delta t} u_{x}^{n-1 / 2}-\frac{2 e \Delta t}{m(2+v \Delta t)} E_{x}^{n},
\end{gathered}
$$

$$
\begin{aligned}
& u_{y}^{n+1 / 2}=\frac{2-v \Delta t}{2+v \Delta t} u_{y}^{n-1 / 2}-\frac{2 e \Delta t}{m(2+v \Delta t)} E_{y}^{n} \\
& u_{z}^{n+1 / 2}=\frac{2-v \Delta t}{2+v \Delta t} u_{z}^{n-1 / 2}-\frac{2 e \Delta t}{m(2+v \Delta t)} E_{z}^{n} .
\end{aligned}
$$

These equations are used for the analysis. The perfectly matched layer (PML) absorbing boundary conditions are applied to truncate the computational domain.

\section{Results and Discussion}

Figures 2(a) and 2(b) show the side view and cross section, respectively, of a ridged-circular nano-aperture. Silver, which has a complex refractive index $N=0.07-4.2 j$ and a thickness $t=150 \mathrm{~nm}$, is assumed to be the metallic material used. The plane wave with a wavelength $\lambda=650 \mathrm{~nm}$, whose electric field is polarized in the $x$ direction, is illuminated vertically to the aperture. In the FDTD analysis, the $5 \mathrm{~nm}$ cubic cells are used. The near-field electric intensity when $g=100 \mathrm{~nm}$ (circular aperture) and $30 \mathrm{~nm}$ is shown in Figures 3(a) and 3(b), respectively. The near-field is observed on the plane $15 \mathrm{~nm}$ below the bottom surface of the aperture as shown in Figure 2(a). The intensity is normalized by the maximum value of the circular nano-aperture shown in Figure 3(a). It is understood that the higher intensity and the smaller spot size can be obtained by using the ridged aperture.

The maximum intensity and full-width half-maximum (FWHM) as a function of $g$ are shown in Figures 4(a) and 4(b), respectively. The maximum intensity has the highest 


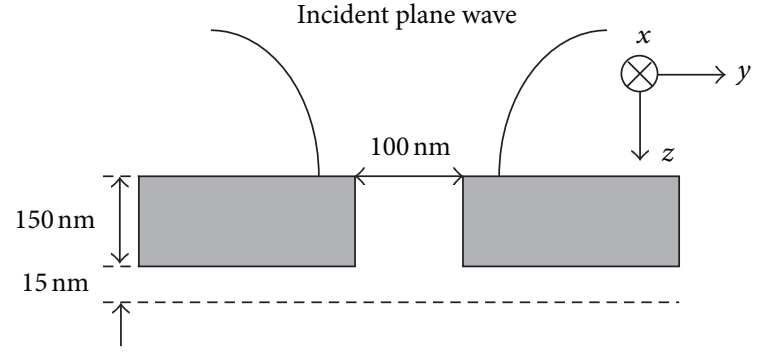

(a)

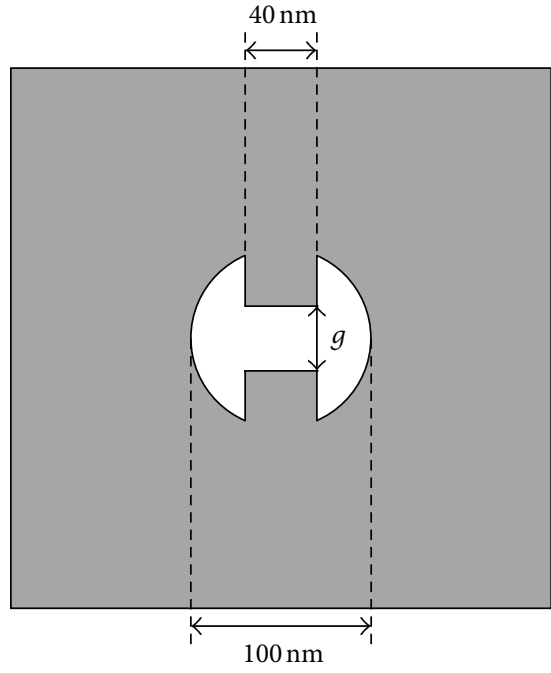

(b)

FIGURE 2: Ridged-circular nanoaperture (a) side view and (b) cross section.

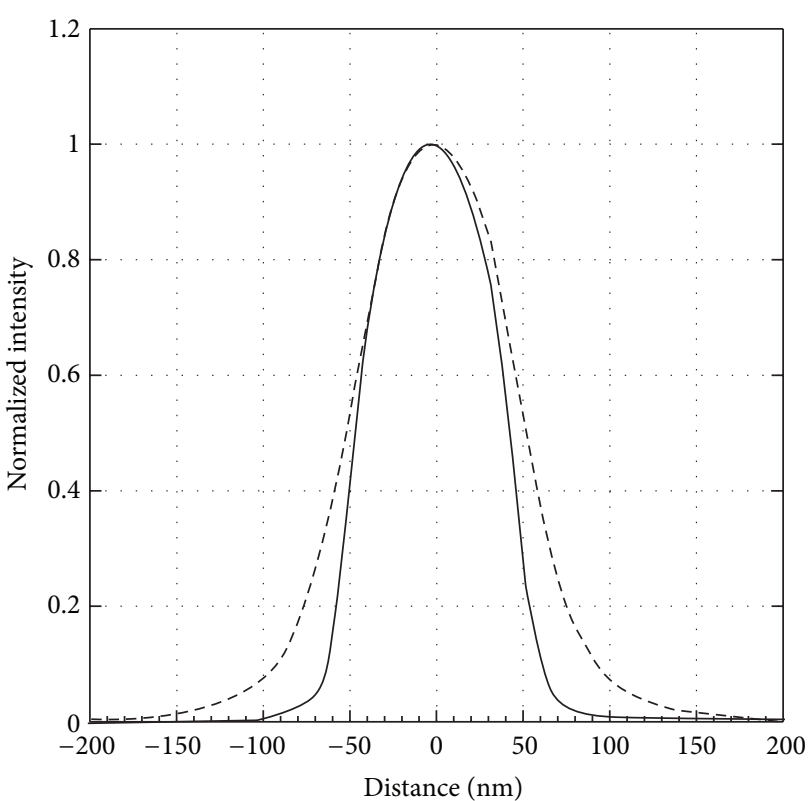

- Along $x$-axis - - Along $y$-axis

(a)

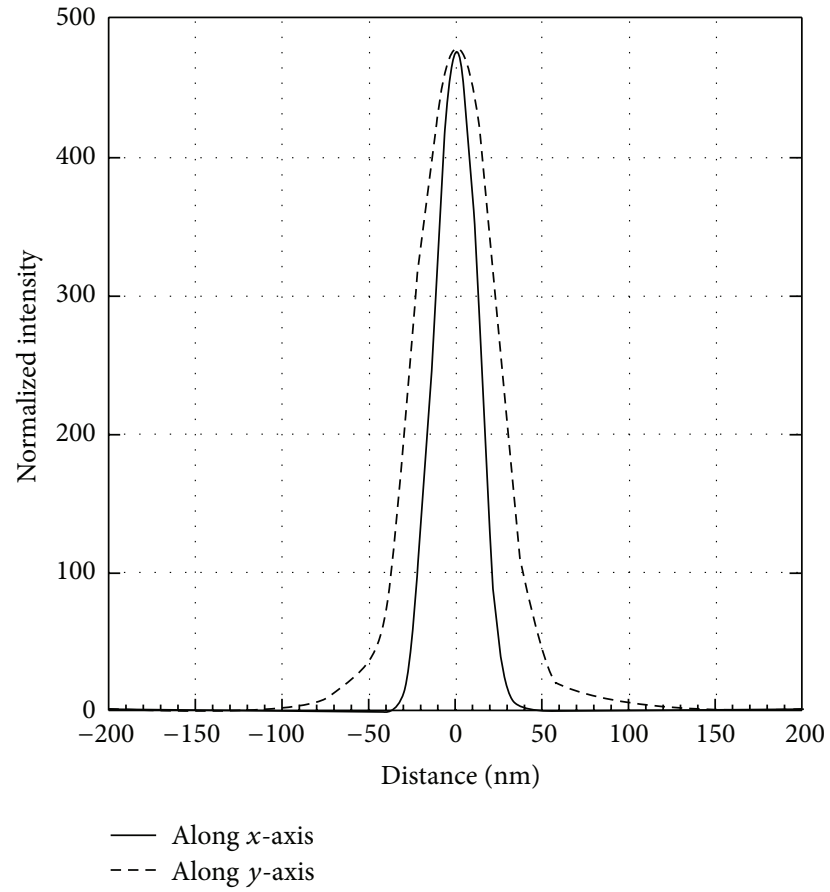

(b)

FIGURE 3: Near-field intensity: (a) circular nanoaperture and (b) ridged-circular nanoaperture ( $g=30 \mathrm{~nm})$.

value when the gap $g$ is about $30 \mathrm{~nm}$ as shown in Figure 4(a). It is understood from Figure 4(b) that the FWHM becomes smaller when using the smaller gap.

Figure 5 shows the structure of a near-field optical disk that consists of a metallic nano-aperture, a phase change disk, two protective films, and a substrate. Here, the ridged-circular nano-aperture shown in Figure 2(b) is used. The refractive index of the phase change disk is $4.6-4.2 j$ (crystalline) and the center of the phase change disk is assumed to be the cylindrical recorded mark with a diameter of $w_{R}$, a thickness of $15 \mathrm{~nm}$, and a refractive index of $4.2-1.9 j$ (amorphous). The refractive indexes of the protective films and the substrate are assumed to be 2.2 and 1.5, respectively. The numerical aperture of the objective lens NA is assumed to be 0.6. The incident light is a Gaussian beam polarized in the $x$ direction whose electromagnetic field components in the frequency 


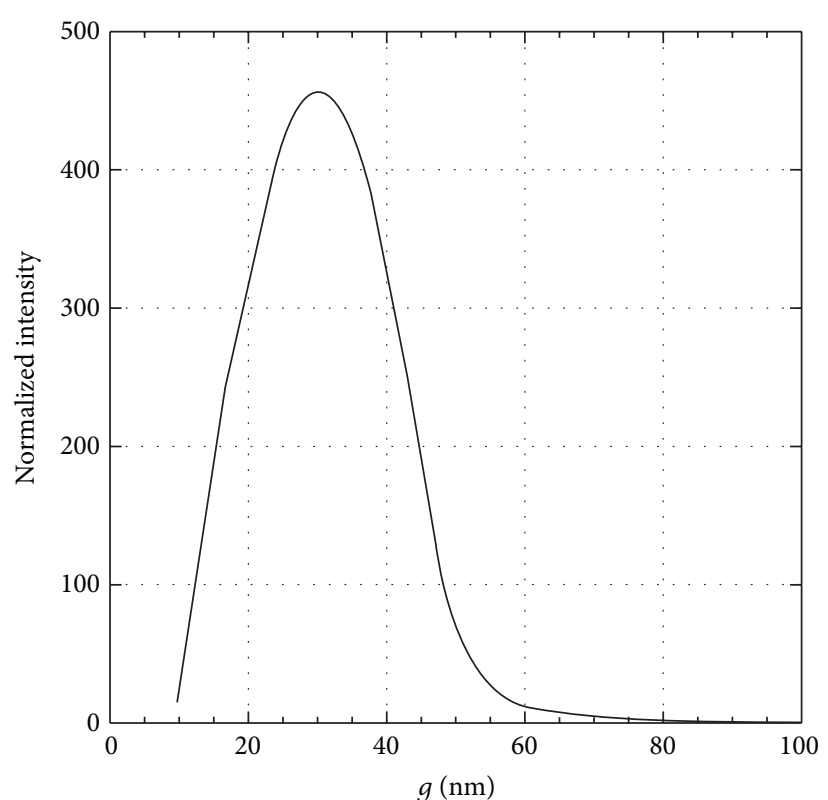

(a)

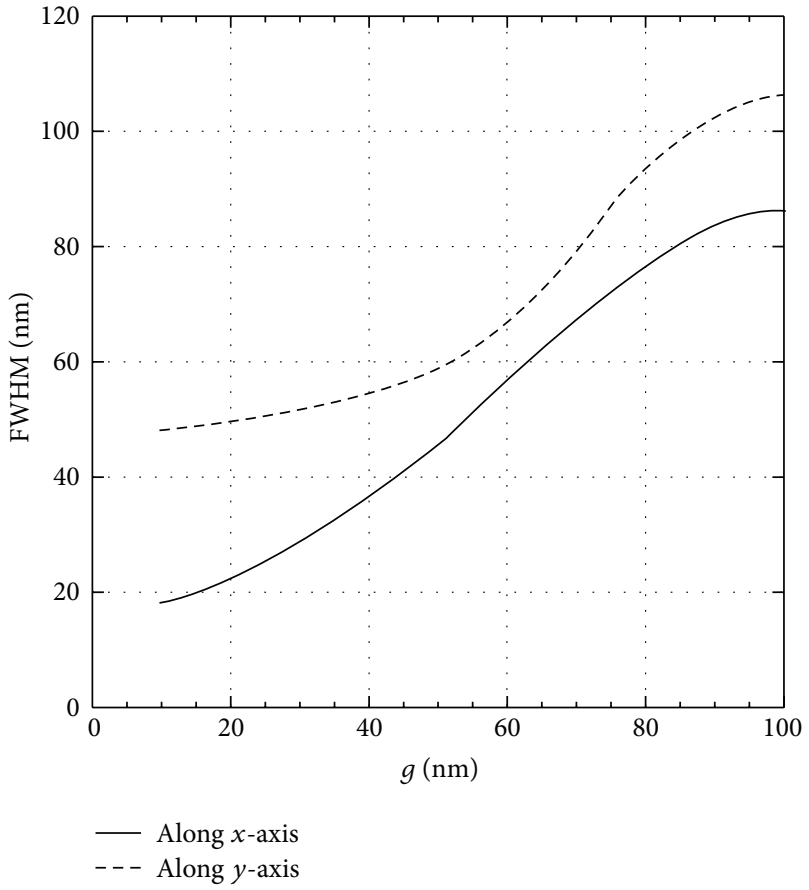

(b)

FIgURE 4: Near-field characteristics as a function of $g$ (a) maximum intensity and (b) FWHM.

domain are expressed as follows when the focus plane is $z=$ $z_{0}$ and the center of the light beam is $\left(x_{0}, y_{0}\right)[8]$ :

$$
\begin{aligned}
& E_{x}^{\mathrm{inc}}= \frac{W_{0}}{W(z)} \exp \\
& \times\left[j\left\{k\left(z-z_{0}\right)+\frac{k r^{2}}{2 R(z)}-\theta(z)\right\}-\frac{r^{2}}{W(z)^{2}}\right], \\
& H_{y}^{\mathrm{inc}}=-\frac{1}{j \omega \mu_{0}} \frac{\partial E_{x}^{\mathrm{inc}}}{\partial z},
\end{aligned}
$$

where $r, W(z), R(z)$, and $\theta(z)$ are written as

$$
\begin{gathered}
r=\sqrt{\left(x-x_{0}\right)^{2}+\left(y-y_{0}\right)^{2},} \\
W(z)=W_{0} \sqrt{1+\left\{\frac{2\left(z-z_{0}\right)}{k W_{0}^{2}}\right\}^{2}}, \\
R(z)=\left(z-z_{0}\right)\left[1+\left\{\frac{k W_{0}^{2}}{2\left(z-z_{0}\right)}\right\}^{2}\right], \\
\theta(z)=\tan ^{-1}\left\{\frac{2\left(z-z_{0}\right)}{k W_{0}^{2}}\right\} .
\end{gathered}
$$

Here, the wavelength $\lambda$ is $650 \mathrm{~nm}$, and $k(=2 \pi / \lambda)$ and $2 W_{0}(=4 /(k N A) \cong 1.06 \lambda)$ are the wave number and the spot size of the light beam, respectively. In this analysis, the focus plane is set on the upper plane of the metallic film shown in Figure 5, and the center of the light beam is equal to that of the aperture.
Figures 6(a) and 6(b) show the far-field scattering patterns of the transmitted wave from the phase change disk in the $x z$ and $y z$ planes, respectively. In these figures, the solid and dashed lines show the results when $w_{R}=100$ and $0 \mathrm{~nm}$ (nonrecorded mark), respectively, and they are normalized by using the maximum value in the $x z$ plane when $w_{R}=100 \mathrm{~nm}$. Here, the transmitted wave is shown because the reflected farfield barely changes when varying the recorded-mark size. It is understood that the light beam, whose electric field is polarized in the $x$ direction, is scattered more widely in the $x$ direction.

Next, the crosstalk characteristics between plural marks are evaluated. Figures 7(a) and 7(b) show the arrangements of three neighboring recorded marks along the $x$ and $y$ axes, respectively. Here, the ridged-circular nano-aperture $(g=$ $30 \mathrm{~nm}$ ) was used. The diameters of the recorded marks are equally $100 \mathrm{~nm}$ and the distances between the marks $\left(d_{x}, d_{y}\right)$ are the parameters.

Figures $8(\mathrm{a})$ and $8(\mathrm{~b})$ show the normalized output as a function of $d_{x}$ and $d_{y}$, respectively. The sum-signal output $\left(I_{\text {sum }}\right)$ was calculated as follows and normalized with the output of the single nonrecorded mark:

$$
I_{\text {sum }}=\int_{0}^{\alpha} \sin \theta \int_{0}^{2 \pi}\left(\left|E_{\phi}^{\mathrm{far}}\right|^{2}+\left|E_{\theta}^{\mathrm{far}}\right|^{2}\right) d \phi d \theta
$$

where $\phi$ is the angle from the positive $x$ axis in the $x y$ plane, $\theta$ is the one from the positive $z$ asis, and $E_{\phi}^{\mathrm{far}}$ and $E_{\theta}^{\mathrm{far}}$ are $\phi$ and $\theta$ components, respectively, of the far-zone transmitted 


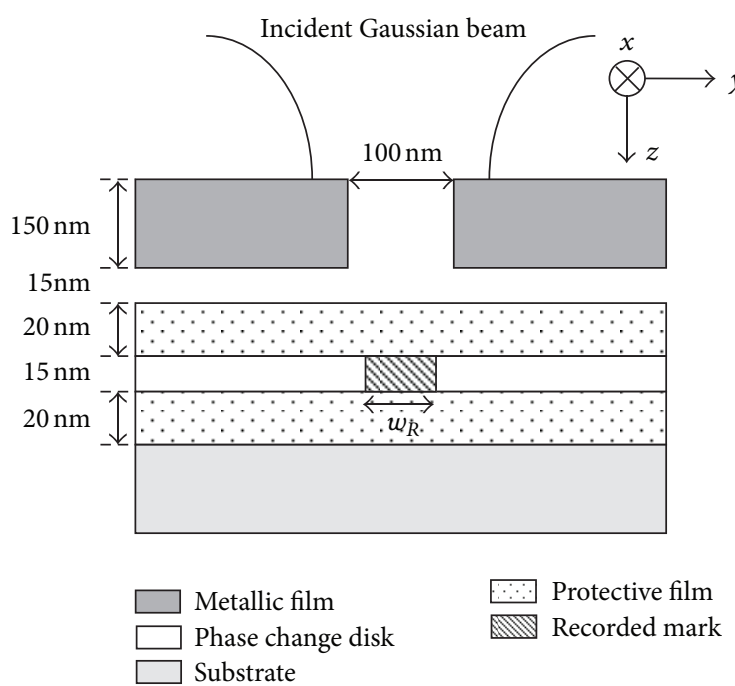

Figure 5: Disk structure.

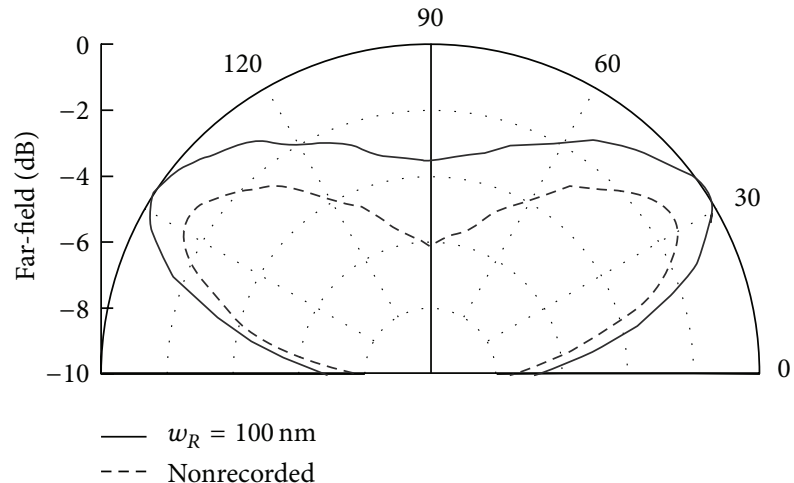

(a)

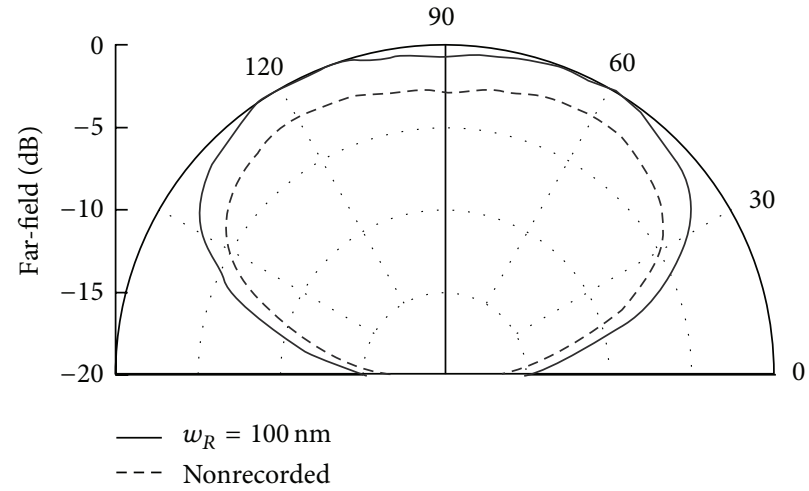

(b)

FIgURE 6: Far-field scattering patterns of transmitted wave (a) $x z$ plane and (b) $y z$ plane.

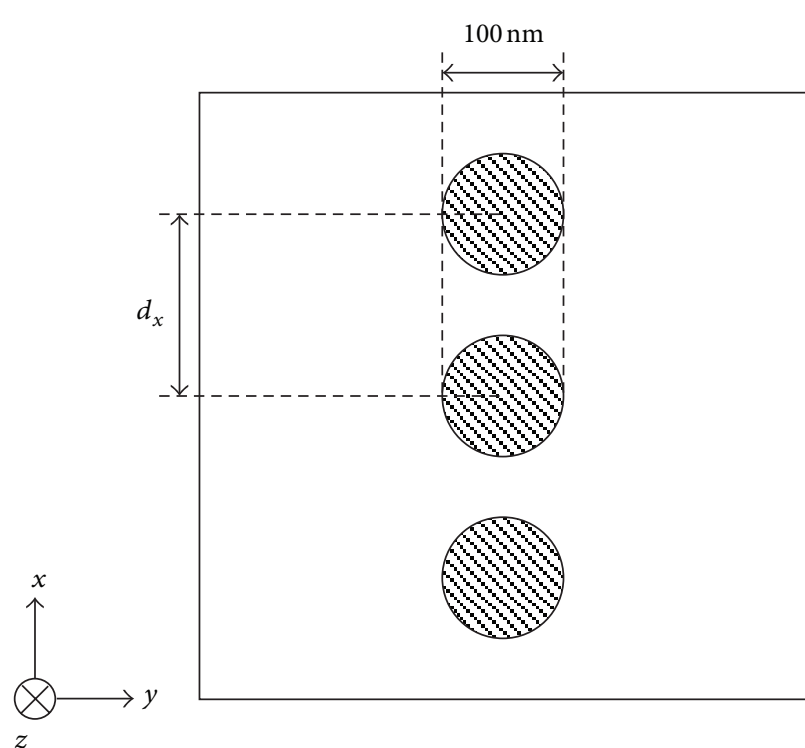

(a)

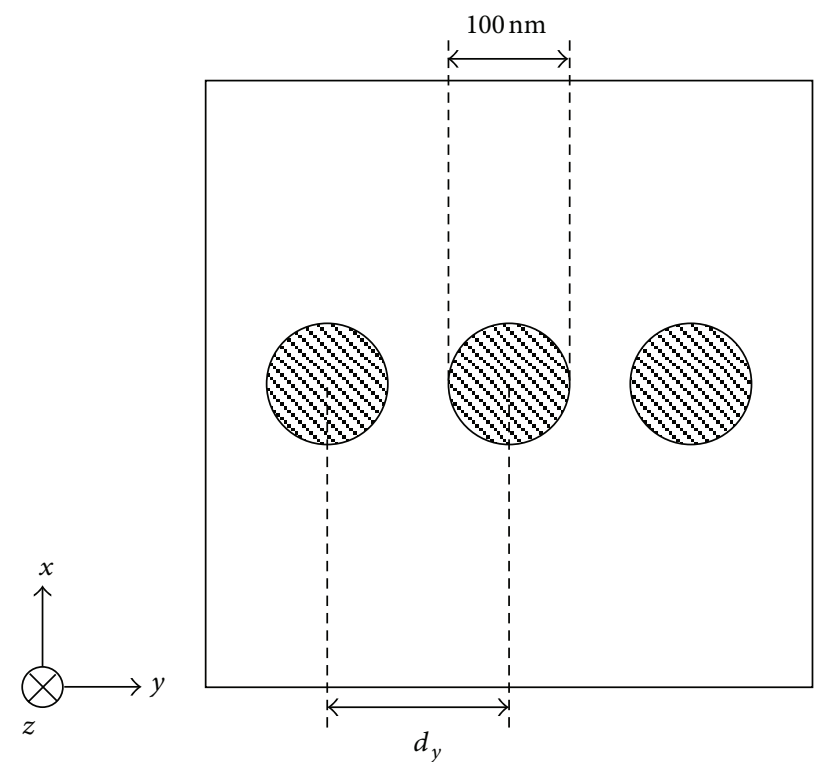

(b)

FIgURE 7: Plural recorded marks (a) along $x$ axis and (b) along $y$ axis. 


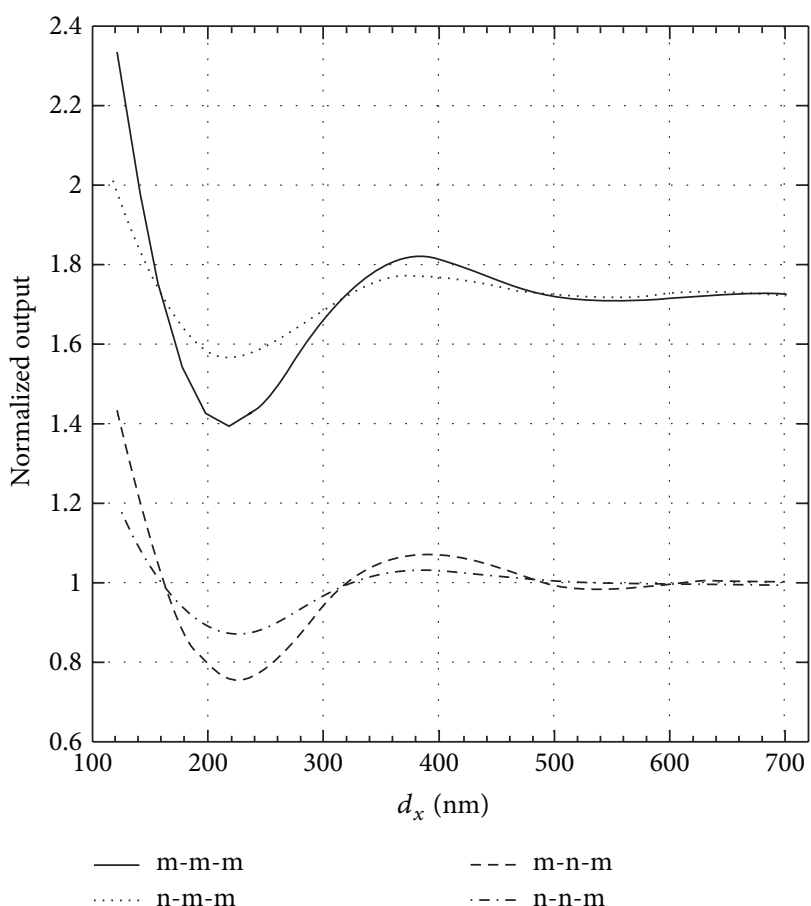

(a)

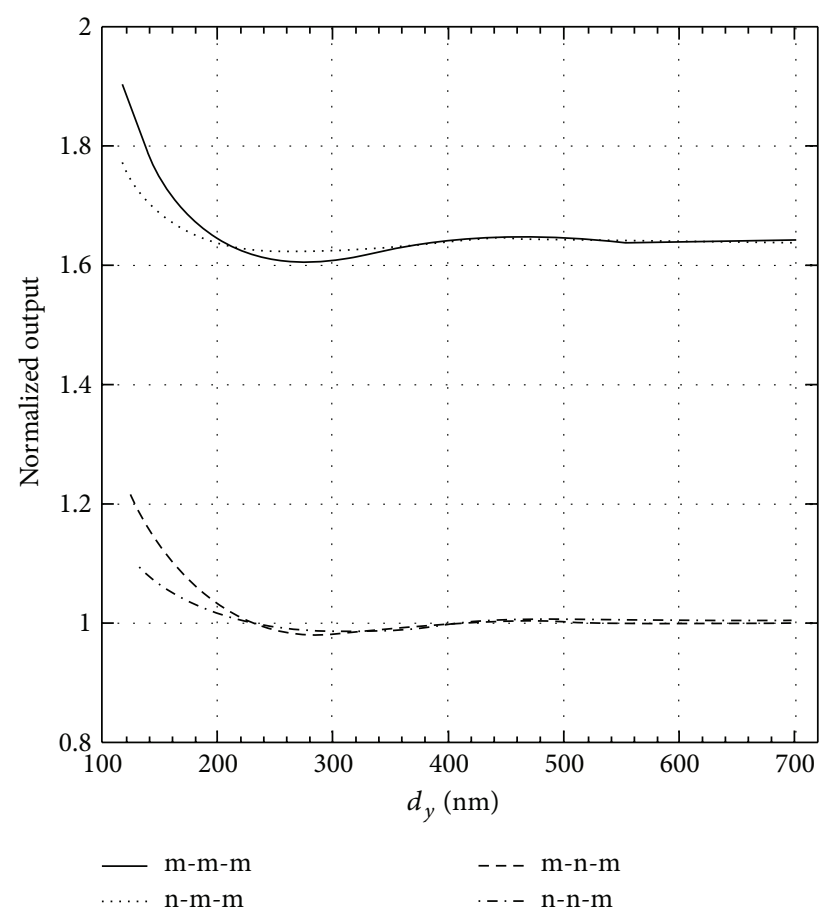

(b)

Figure 8: Crosstalk characteristics (a) along $x$ axis and (b) along $y$ axis.

electric field in the spherical coordinate. Here, $\alpha$ is calculated as

$$
\alpha=\sin ^{-1}\left(\frac{\mathrm{NA}}{n_{\mathrm{sub}}}\right),
$$

where $n_{\text {sub }}$ is the refractive index of the substrate $\left(n_{\text {sub }}=1.5\right)$.

The arrangements of the three neighboring marks are denoted by $m-m-m, n-m-m, m-n-m$, and $n-n-m$, where " $m$ " and " $n$ " represent the recorded and nonrecorded marks, respectively. Along the $x$ axis the influence of the neighboring marks exists when $d_{x}$ is much larger than the recorded-mark size. On the other hand, the variation in output with regard to $d_{y}$ is smaller.

\section{Conclusion}

The ridged-circular nanoaperture for a near-field optical disk was analyzed by using the FDTD method into which the motion equations of free electrons were inserted. The near-field characteristics of the aperture were investigated. The phase change disk illuminated by the optical near-field through a ridged-circular nano-aperture was also analyzed and the crosstalk characteristics between the plural marks were shown.

\section{References}

[1] S. Omodani, T. Saiki, and M. Obara, "Metallic slit aperture as a near-field optical head for heat-assisted magnetic recording," Journal of Applied Physics, vol. 105, no. 1, Article ID 013101, 2009.
[2] Y.-M. Wu, L.-W. Li, and B. Liu, "Gold bow-tie shaped aperture nanoantenna: Wide band near-field resonance and far-field radiation," IEEE Transactions on Magnetics, vol. 46, no. 6, pp. 1918-1921, 2010.

[3] L. Scorrano, F. Bilotti, and L. Vegni, "Achieving power transmission enhancement by using nano-rings made of silver spheres," IEEE Photonics Technology Letters, vol. 22, no. 21, pp. 1595-1597, 2010.

[4] K. Nakagawa, Y. Ashizawa, S. Ohnuki, A. Itoh, and A. Tsukamoto, "Confined circularly polarized light generated by nano-size aperture for high density all-optical magnetic recording," Journal of Applied Physics, vol. 109, no. 7, Article ID 07B735, 2011.

[5] S. Kagawa, Y. He, and O. Kojima, "Two-dimensional FDTD analysis of the readout characteristics of an optical near field disk," IEICE Transactions on Electronics, vol. E91-C, no. 1, pp. 48-55, 2008.

[6] T. Kitamura and S. Iwata, "Analysis of a near-field optical disk with an acute-edged metallic nano-aperture," IEICE Transactions on Electronics, vol. E93-C, no. 9, pp. 1474-1477, 2010.

[7] T. Kitamura, "FDTD analysis of a near-field optical disk with a ridged-square nano-apertre," IEICE Transactions on Electronics, vol. E95-C, no. 6, pp. 1110-1116, 2012.

[8] Y. He, T. Kojima, T. Uno, and S. Adachi, "FDTD analysis of three-dimensional light-beam scattering from the magnetooptical disk structure," IEICE Transactions on Electronics, vol. E81-C, no. 12, pp. 1881-1887, 1998. 

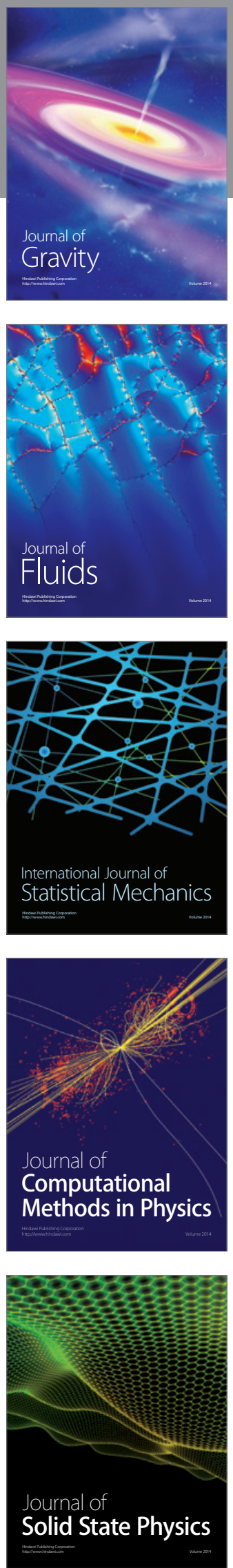

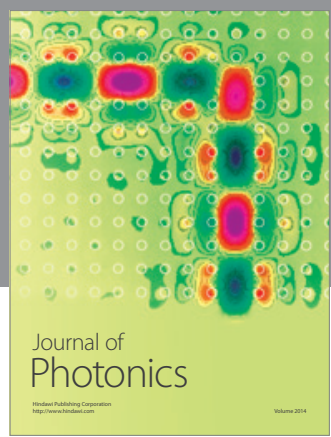

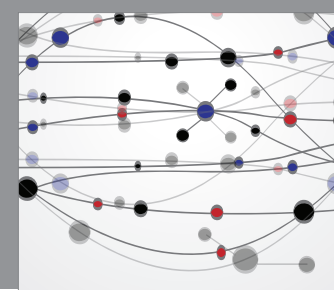

The Scientific World Journal

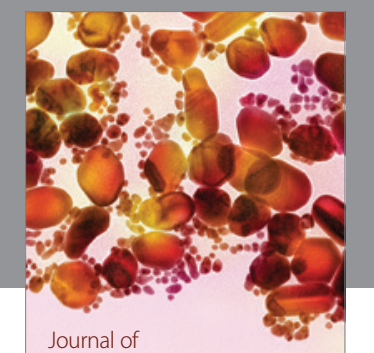

Soft Matter
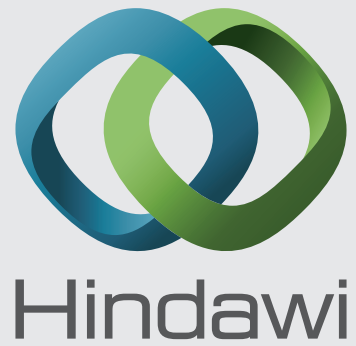

Submit your manuscripts at

http://www.hindawi.com
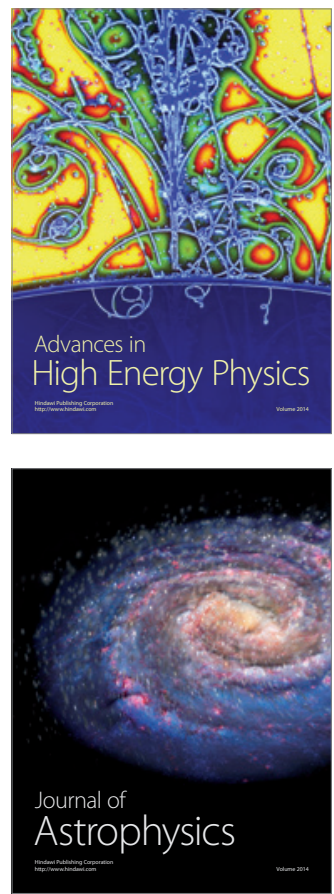
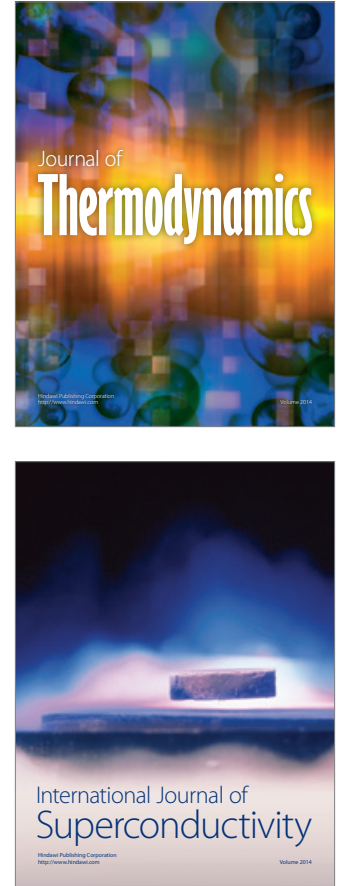
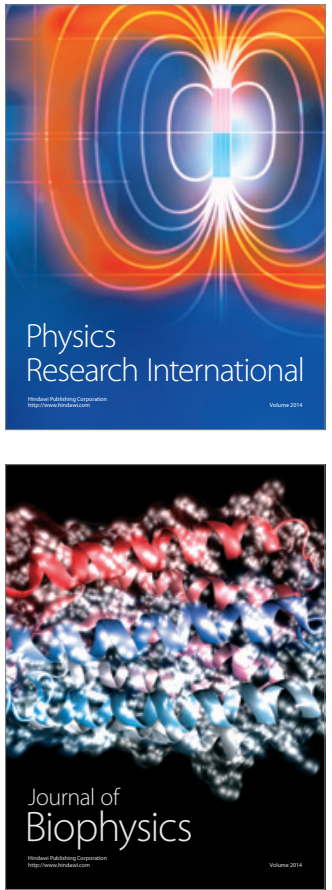
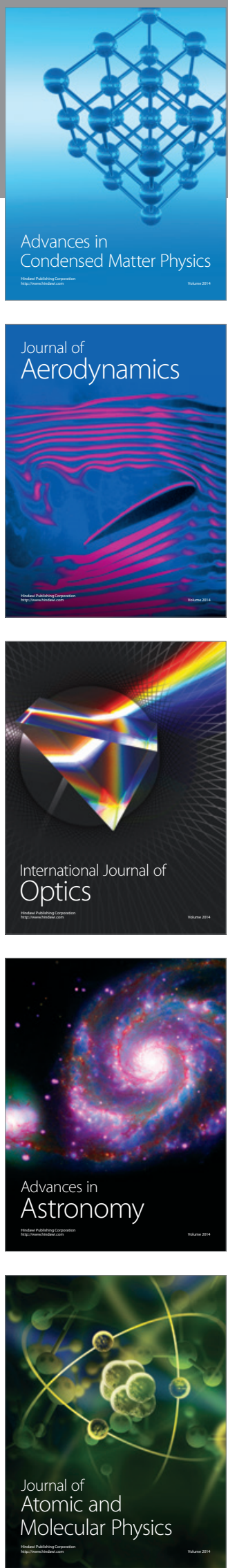